\title{
Transcatheter aortic valve implantation in the presence of an anomalous left circumflex coronary artery: a case report
}

\author{
Mackenzie Mbai $^{1,2}$, Alok Sharma ${ }^{1,2}$, Brett Oestreich ${ }^{2}$, Asher Sobotka ${ }^{3}$, Rosemary F. Kelly ${ }^{1,2}$, Horst Sievert ${ }^{3}$, \\ Stefan Bertog ${ }^{1,3}$ \\ ${ }^{1}$ Minneapolis Veterans Affairs Medical Center, Minneapolis, MN, USA; ${ }^{2}$ University of Minnesota, Minneapolis, MN, USA; ${ }^{3}$ CardioVascular Center \\ Frankfurt, Frankfurt, Germany \\ Correspondence to: Stefan Bertog, MD. One Veterans Drive, Minneapolis, MN 55417, USA. Email: sbertog@aol.com.
}

\begin{abstract}
This is a case of an anomalous left circumflex coronary artery (LCX) originating from the right coronary cusp with a retroaortic course in a patient with severe aortic valve stenosis requiring transcatheter aortic valve implantation (TAVI). In the context of TAVI, an anomalous LCX has been described only in a few case reports. In some, the LCX remains unaffected. However, occlusion after TAVI valve deployment has been described. We report pre-procedural imaging and procedural precautions prior to valve deployment in our case in which the LCX remained unaffected by the TAVI valve. More case reports or series are needed to help assess which characteristics are associated with an increased risk of anomalous LCX obstruction.
\end{abstract}

Keywords: Transcatheter aortic valve implantation (TAVI); transcatheter aortic valve replacement (TAVR); anomalous coronary artery; anomalous left circumflex coronary artery (LCX); coronary protection; case report

Submitted Dec 29, 2019. Accepted for publication Feb 07, 2020.

doi: $10.21037 / \mathrm{cdt} .2020 .03 .05$

View this article at: http://dx.doi.org/10.21037/cdt.2020.03.05

\section{Introduction}

Data regarding the safety of transcatheter aortic valve implantation (TAVI) in patients with an anomalous left circumflex coronary artery (LCX) originating from the right coronary cusp with a retro-aortic course are limited. Some have reported LCX occlusion (1) while in other cases the LCX remained unaffected (2). We describe the case of a 72 -year-old male with severe aortic stenosis (Sievers I bicuspid aortic valve with fusion of the right and left coronary cusp) (Video 1) and low surgical risk for surgical aortic valve replacement. Pre-procedural workup revealed an anomalous LCX from the right coronary cusp with retro-aortic course in immediate proximity to the aortic valve annulus (Figures 1,2 and Videos 1-3). As part of our heart team approach, the patient was reviewed in our combined Cardiology/Cardiovascular Surgery conference and the possibility of LCX occlusion by compression via the TAVI valve was entertained. However, taking into account that there is also a risk of coronary occlusion during surgical aortic valve replacement, we favored TAVI using coronary protection during valve deployment.

\section{Procedure}

The patient provided written informed consent for TAVI. The procedure was performed via transfemoral approach. A Sentinel device (Boston Scientific, Marlborough, MA, USA) was used for cerebral protection via the right radial artery and a $6 \mathrm{~F} \mathrm{JR}-4$ guide catheter (via transfemoral access) was used to engage the anomalous LCX. The LCX was wired without difficulty with a 0.014 inch Luge wire (Boston Scientific, Marlborough, MA, USA) and a $6 \mathrm{~F}$ guideliner catheter (Vascular Solutions, Minneapolis, $M N$, USA) was advanced into the mid to distal anomalous LCX over the Luge wire. The guideliner catheter was used to allow disengagement of the larger coronary guide catheter during valve deployment and potentially prevent guide catheter induced right coronary ostial injury by interference of the guide catheter and valve stent frame. Further, the guideliner could potentially allow more support and stability of coronary equipment (wire and 
stent) during valve delivery and deployment. Then a $3.5 \mathrm{~mm} \times 38 \mathrm{~mm}$ drug eluting stent was positioned within the guideliner catheter in the proximal anomalous LCX (Figure 3). The guideliner catheter was left in place

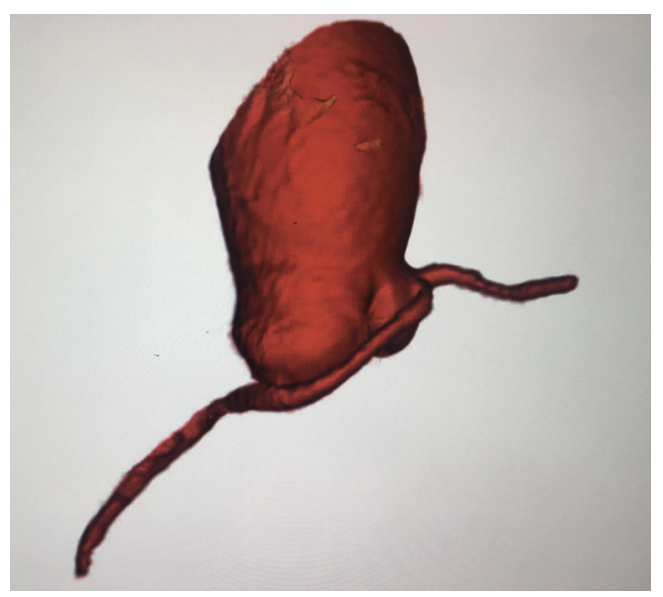

Figure 1 This illustrates the retroaortic course of the anomalous left circumflex coronary artery in immediate proximity to the aortic valve annulus. while the JR-4 guide catheter was withdrawn slightly during TAVI valve deployment. A $26 \mathrm{~mm}$ Sapien S3 valve (Edwards Life Sciences, Irvine, CA, USA) was then deployed at nominal inflation volume (Video 4). Thereafter, a cineangiogram of the anomalous LCX was performed that demonstrated a widely patent LCX (Figure 4 and Video 5) in two orthogonal views. The coronary guidewire/stent and guide catheter were subsequently removed. There was no aortic insufficiency (Video 6) or relevant transvalvar gradient after valve deployment.

\section{Discussion}

There are only very few reports of TAVI in the context of an anomalous LCX artery. While our case, similar to other cases, illustrates that TAVI can be performed safely without compromising the anomalous LCX despite immediate proximity to the aortic annulus, there is one case report that demonstrates acute coronary occlusion of an anomalous LCX during TAVI with a balloon expandable (Sapien XT) valve (1). It remains unclear why some cases do not appear to impact the anomalous LCX while others do. Ujihira et al.

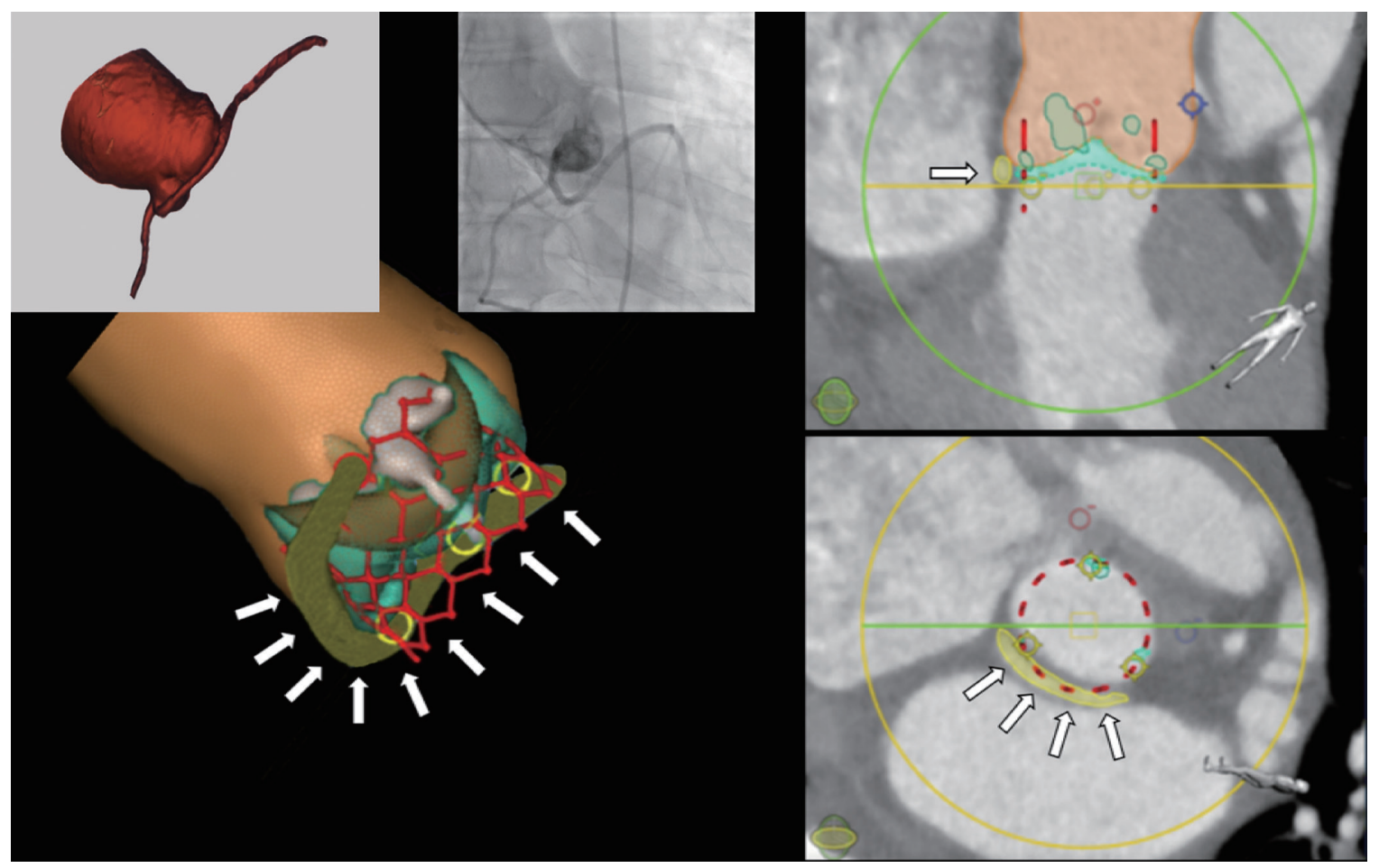

Figure 2 This illustrates a simulated Sapien S3 valve in the planned position as well as the anomalous left circumflex coronary artery (green, white arrows). 


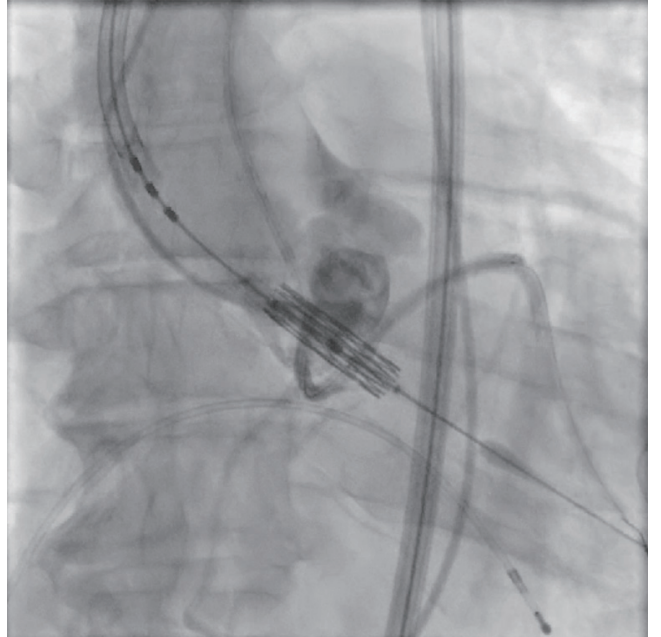

Figure 3 Aortic root angiography demonstrating the undeployed Sapien S3 valve in addition to the guideliner catheter and guide wire as well as stent in the anomalous left circumflex coronary artery and the guide catheter disengaged.

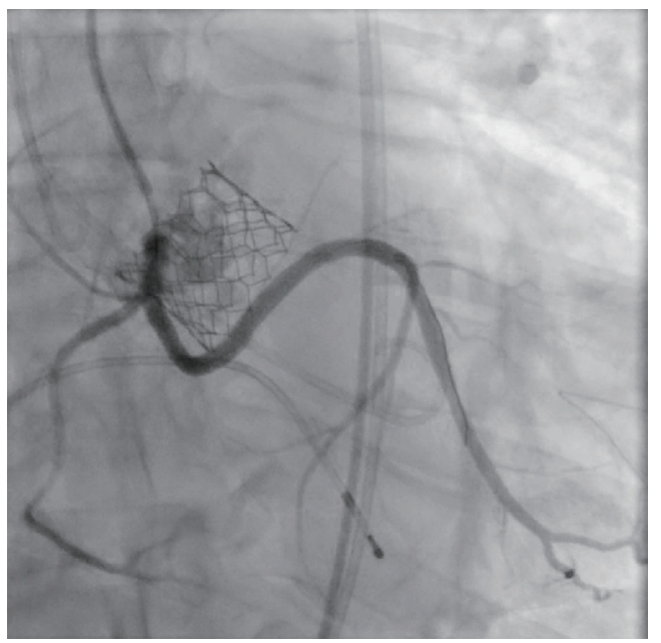

Figure 4 The Sapien S3 valve has been deployed and the guideliner catheter as well as stent removed with final selective angiography of the anomalous left circumflex artery.

report three cases of an anomalous LCX with a retro-aortic course without coronary compression (a balloon-expandable Sapien 3 valve was used for all three cases) (2). All patients were elderly and coronary calcium was noted on computed tomography (CT). It was proposed that the coronary calcium may have had a protective effect. However, our case shows that there appear to be more factors that determine the behavior of an anomalous LCX during TAVI because, in our case example, there was no coronary calcium and valve calcium was absent at the level of the annulus in proximity to the LCX. In addition to the coronary calcium described by Ujihira et al., another feature unique to the cases without compression of the anomalous LCX appears to be the level of the retro-aortic course of the LCX. The images in the manuscript provided by authors who demonstrated an unaffected anomalous LCX after TAVI suggest a retro-aortic anomalous LCX course at the level of the leaflets rather than the annulus which may render LCX compression less likely. Likewise, Cho et al. describe a case of an anomalous LCX that was not protected during TAVI (with a balloon expandable Sapien 3 valve) because the level of its course was at a safe distance from the annulus (3). One unique feature of the case of anomalous LCX compression causing coronary occlusion was $20 \%$ oversizing of the balloon expandable valve which may have increased the risk of compression (1). Regardless, in the absence of more anatomical data surrounding TAVI cases with an anomalous LCX, the mechanism of coronary occlusion and protective or risk factors remain to be determined and it appears prudent to adopt an approach of coronary protection during TAVI in these cases. Of note, it is not clear whether the use of a self-expanding Evolut valve (Medtronic, Dublin, Ireland) or mechanically expanding Lotus valve (Boston Scientific, Marlborough, MA, USA) would be less likely to cause coronary occlusion. In fact, the size of the inflow of the recommended $29 \mathrm{~mm}$ Evolut valve at the level of the annulus would have been larger than the size of the $26 \mathrm{~mm}$ Sapien valve and, hence, anomalous coronary compression may have been more likely with the self-expanding Evolut valve. Though both, the self-expanding Evolut and mechanically expanding Lotus value are retrievable in case coronary occlusion occurs, it should be recognized that the nitinol of the self-expanding valve cage may continue to expand and could cause delayed coronary compromise after the procedure at which time the coronary is no longer protected.

\section{Conclusions}

Limited data are available regarding the risk and risk factors of occlusion of an anomalous LCX artery with a course in immediate proximity to the aortic valve annulus after TAVI. In the absence of organized data to predict procedure related occlusion and or risk factors, it appears prudent to perform extensive pre-procedural imaging analysis to better understand the anatomy and also, to protect the artery intra-procedurally. 


\section{Acknowledgments}

Funding: None.

\section{Footnote}

Conflicts of Interest: All authors have completed the ICMJE uniform disclosure form (available at http://dx.doi. org/10.21037/cdt.2020.03.05). SB serves as an unpaid editorial board member of Cardiovascular Diagnosis and Therapy from Jul 2019 to Jun 2021. The other authors have no conflicts of interest to declare.

Ethical Statement: The authors are accountable for all aspects of the work in ensuring that questions related to the accuracy or integrity of any part of the work are appropriately investigated and resolved. Written informed consent was obtained from the patient for publication of this manuscript and any accompanying images.

Open Access Statement: This is an Open Access article distributed in accordance with the Creative Commons Attribution-NonCommercial-NoDerivs 4.0 International License (CC BY-NC-ND 4.0), which permits the noncommercial replication and distribution of the article with the strict proviso that no changes or edits are made and the original work is properly cited (including links to both the formal publication through the relevant DOI and the license). See: https://creativecommons.org/licenses/by-nc-nd/4.0/.

\section{References}

1. Acosta-Vélez JG, García del Blanco B, Guindo J, et al. Acute artery occlusion during transcatheter aortic valve replacement in a patient with an anomalous origin of the circumflex artery. JACC Cardiovasc Interv 2014;7:1324-5

2. Ujihira K, Raval AN, Wolff MR, Osaki S. Transcatheter aortic valve replacement in patients with anomalous left circumflex coronary artery. J Card Surg 2019;34:503-5.

3. Cho SW, Kim BG, Park TK et al. Transcatheter aortic valve replacement in a patient with anomalous origin of the left coronary artery. J Cardiol Cases 2019;19:133-5.

Cite this article as: Mbai M, Sharma A, Oestreich B, Sobotka A, Kelly RF, Sievert H, Bertog S. Transcatheter aortic valve implantation in the presence of an anomalous left circumflex coronary artery: a case report. Cardiovasc Diagn Ther 2020;10(2):223-226. doi: $10.21037 /$ cdt.2020.03.05 